experimental, and clinical (case-control) studies were excluded. Reference lists of relevant papers were scanned for any additional articles of interest. Data extraction and methodological quality assessment using the STROBE checklist were conducted independently by two raters. Multi-level meta-analyses were conducted, with consideration of a number of potential moderators, including mean age of sample at baseline, length of follow-up, and quality of study.

Results After removal of duplicate references, all papers were screened for eligibility using a three-step process: 1) title screening $(n=20,954) ; 2)$ abstract screening $(n=981)$; and 3$)$ full text screening $(n=172)$. Inter-rater reliability at each stage of screening was $>90 \%$. A total of 17 studies (with 8 assessing depression as a binary variable and 9 assessing depression as a continuous variable) met eligibility criteria and had sufficient statistical information for extraction. The results of the meta-analyses will be presented and discussed with a focus on the effects of the key moderators that may influence the link between depression and memory decline, such as mean age at baseline, length of follow-up, and quality of study. Preliminary analyses suggest that affective problems significantly increase risk for subsequent decline in memory (Binary meta-analysis: $\mathrm{OR}=1.47 ; 95 \% \mathrm{CIs}=1.15,1.87, \mathrm{p}=0.002$; Continuous metaanalysis: $\quad \mathrm{B}=-0.007, \quad 95 \% \quad \mathrm{CIs}=-0.011,-0.002, \mathrm{p}=0.003)$. Conclusions Results of the present study improve current understanding of the temporal nature of the association between depression and memory across the life-course. This has important implications for the identification of individuals who are at a particularly high risk for accelerated decline in memory function and dementia.

\section{P10 DIET QUALITY, SARCOPENIA AND FRAILTY IN OLDER MEN: CROSS SECTIONAL ANALYSIS FROM THE BRITISH REGIONAL HEART STUDY}

${ }^{1} \mathrm{TJ}$ Parsons*, ${ }^{2} \mathrm{E}$ Papachristou, ${ }^{3}$ SE Ramsay, ${ }^{4} \mathrm{JL}$ Atkins, ${ }^{1} \mathrm{O}$ Papacosta, ${ }^{1} \mathrm{LT}$ Lennon, ${ }^{1} \mathrm{~S}$ Ash, ${ }^{5} \mathrm{PH}$ Whincup, ' $\mathrm{SG}$ Wannamethee. ${ }^{1}$ Primary Care and Population Health, UCL, London, UK; ${ }^{2}$ Institute of Education, UCL, London, UK; ${ }^{3}$ Institute of Health and Society, Newcastle University, Newcastle, UK; ${ }^{4}$ Epidemiology and Public Health, University of Exeter, Exeter, UK; ${ }^{5}$ Population Health Research Institute, St George's University of London, London, UK

\subsection{6/jech-2017-SSMAbstracts.112}

Background Frailty, a vulnerability to adverse health outcomes, and sarcopenia, a decline in muscle mass and strength or performance are associated with ageing. Frailty and sarcopenia predict increased mortality and hospitalisation, and sarcopenia often occurs with an increase in body fat known as sarcopenic obesity which elevates these risks further. Diet quality is well established as a predictor of mortality, but few studies have investigated diet quality in relation to frailty or sarcopenia, and findings are inconclusive. We have therefore examined the associations between diet quality, frailty and sarcopenic obesity categories.

Methods We used cross sectional data from community-dwelling men aged 71-91 years (British Regional Heart Study) in 2010-2012 recruited from 24 primary care practices. Men completed a food frequency questionnaire, from which the Healthy Dietary Index (HDI) and Elderly Dietary Index (EDI) were derived, and attended a physical examination. Frailty was based on the 5 components of the Fried frailty phenotype and we used a sarcopenic obesity classification which defines 4 groups; optimal, sarcopenic, obese or sarcopenic obese based on waist circumference and mid-arm muscle circumference.
We used logistic regression models to investigate whether diet quality was associated with frailty and sarcopenia and/or obesity.

Results $1331 / 3137$ men (42\%) had data for sarcopenia/obesity, all covariates and diet quality and 1119 men (36\%) for frailty, covariates and diet quality. After adjusting for age, social class, region of residence, smoking, alcohol consumption, cardiovascular disease and energy intake, men in the top quartile of the HDI score had a lower odds of being frail $(0.5895 \% \mathrm{CI}$ $0.34,0.96)$ compared with men in the bottom quartile, and men in the top quartile of either HDI or EDI had a lower odds of being obese compared with men in the bottom quartile $(0.5295 \%$ CI $0.33,0.84 \%$ and 0.57 95\% CI $0.38,0.86$ respectively). Neither the HDI or EDI was associated with sarcopenia or sarcopenic obesity, and the EDI was not associated with frailty.

Conclusion Higher diet quality based on both the HDI and EDI is associated with obesity but we found no evidence that diet quality is associated with sarcopenia in these elderly British men. However, our findings suggest that a higher diet quality as indicated by the HDI, a measure of adherence to WHO nutrient intake guidelines, might be relevant for the prevention or reversal of frailty.

\section{P11 THE ENDURING INFLUENCE OF CONTROLLING PARENTING ON PERSONAL MASTERY IN OLDER AGE}

FH Harkness*, M Stafford, T Cosco, M Richards. 'MRC Unit of Lifelong Health and Ageing, UCL, London, UK; ${ }^{2} M R C$ Unit of Lifelong Health and Ageing, UCL, London, UK; ${ }^{3} M R C$ Unit of Lifelong Health and Ageing, UCL, London, UK; ${ }^{4}$ MRC Unit of Lifelong Health and Ageing, UCL, London, UK

\subsection{6/jech-2017-SSMAbstracts.113}

Background Personal mastery is the subjective feeling of control over the events in one's own life. It is associated with healthy ageing, including better cardio-metabolic health, immune function and physical functioning. As an adult mastery is strongly associated with achievements of education, income and social class. However, within-group differences indicate that there could be other ways to feel in control. Mastery is theorised to be a self-concept first learnt in adolescence, and as such family may play a role in shaping it. Those whose parents support them psychologically and allow them appropriate freedom as an adolescent may grow up perceiving themselves to be in control, over and above tangible socio-economic resources.

Data The Medical Research Council National Survey of Health and Development (NSHD) is a representative sample of births in mainland Britain that occurred during a week in March 1946. Participants were $(n=1,037)$ study members who had provided data at ages $4,26,43$ and 68. Controlling parenting was measured using the Parental Bonding Instrument (PBI). This measures percieved parental levels of psychological control (e.g. invasiveness, overprotection).

The outcome was personal mastery assessed at age 68 using Pearlin's 7 item scale. An example item is, " I have little control over the things that happen to me." Multivariable regression analysis was used to test the association between psychologically controlling parenting and personal mastery age 68, controlling for childhood and adult socio-economic markers.

Results Higher perceived parental psychological control was associated with lower mean mastery -0.12 (95\% $0.20,-0.04$ ) 
aged 68. This association was not attenuated when adjusting for childhood and adult socio-economic position.

Conclusion These findings show the lifelong importance of parenting on psychological self-concepts. Restricting young-life psychological freedom may build an enduring feeling that one is not agent of one's own control. Despite having access to a comfortable socio-economic position, those whose parents were more controlling in adolescence felt less in control of their own life age 68 .

\section{P12 EVALUATING POLICY RESPONSES TO UPSTREAM DETERMINANTS OF CHRONIC, NON-COMMUNICABLE DISEASES: SUPPORTING HEALTHY DIETS AND ACTIVE LIVING IN SEVEN CARIBBEAN COUNTRIES}

\begin{abstract}
${ }^{1}$ MM Murphy* ${ }^{2,3} \mathrm{C}$ Guell, ${ }^{1,4} \mathrm{TA}$ Samuels, ${ }^{1} \mathrm{~L}$ Bishop, ${ }^{3,4} \mathrm{~N}$ Unwin. ${ }^{1}$ Faculty of Medical Sciences, University of the West Indies, Bridgetown, Barbados; ${ }^{2}$ European Centre for Environment and Human Health, University of Exeter Medical School, Truro, UK; ${ }^{3} M R C$ Epidemiology Unit and Centre for Diet and Activity Research, University of Cambridge, Cambridge, UK; ${ }^{4}$ Chronic Disease Research Centre, University of the West Indies, Bridgetown, Barbados
\end{abstract}

\subsection{6/jech-2017-SSMAbstracts. 114}

Background In 2007, heads of government in the Caribbean Community (CARICOM) committed to concerted policy action to address non-communicable diseases, whose burden was recognised as a threat to regional development. In 2015, a large mixed-method evaluation study investigated the progress made in developing and implementing relevant policies. As part of this, a qualitative study in seven Caribbean countries aimed to identify, assess and compare existing policies, gaps in policy responses, and the factors influencing successful policy development and implementation.

Methods Policy document analysis was complemented by 76 semi-structured interviews with 80 relevant stakeholders in government, civil society and the private sector. Data collection and analysis protocols were developed iteratively. Interviews were audio-recorded and analysed pragmatically framed by the WHO NCD Action Plan, a Multiple Streams policy approach and realist evaluation principles. An analysis team coded using Dedoose software, after which two lead researchers synthesised the analyses.

Results Policy gaps existed regarding alcohol, diet and physical activity. Most widely reported successes across countries were policies and health promotion initiatives to support healthy eating in communities and in schools, including the development of dietary guidelines. Physical activity was targeted primarily in schools, with public participation in public sports events. Successful initiatives were often marked by collaboration between government ministries such as health, education and agriculture. There were very few existing policies around alcohol harm. The impact of these initiatives was reported as limited by adverse upstream determinants, including reliance on food imports entwined with the consequences of upholding existing trade agreements, which constrained more impactful fiscal and legislative action to support availability, quality and affordability of healthy foods. There was little evidence of policy responses to create physical and social environments conducive to active living, such as to support greater walking. The lack of focus on policy creation, lack of monitoring and evaluation of existing interventions, and limited staffing and financial capacities limited success in policy implementation. For alcohol specifically, national and political cultural attitudes and its economic importance deemed it untouchable from a policy standpoint.

Conclusion The least well developed policy responses concern upstream determinants of unhealthy diets, physical inactivity and alcohol harm. Local and regional political support is essential to accelerate action to support environments conducive to healthy eating and active living. Addressing reliance on food imports and existing trade agreements as well as developing and implementing policies to reduce alcohol related harm necessitates regional cooperation for a unified response.

\section{P13 SOCIAL, BIOLOGICAL, BEHAVIOURAL AND PSYCHOLOGICAL FACTORS AND PHYSICAL ACTIVITY DURING PREGNANCY: A CROSS-SECTIONAL STUDY}

${ }^{1} \mathrm{C}$ Flannery*, ${ }^{2} \mathrm{D}$ Dahly, ${ }^{1} \mathrm{M}$ Byrne, ${ }^{3}$ Ali Khashan, ${ }^{3} \mathrm{~S}$ McHugh, ${ }^{4} \mathrm{~L}$ Kenny, ${ }^{5} \mathrm{FM}$ McAuliffe, ${ }^{3} \mathrm{PM}$ Kearney. ${ }^{1}$ Health behaviour change research group, National University of Ireland, Galway, Ireland; ${ }^{2}$ HRB Clinical Research Facility Cork, University College Cork, Cork, Ireland; ${ }^{3}$ Department Epidemiology and Public Health, University College Cork, Ireland; ${ }^{4}$ Department Obstetrics and Gynaecology, University College Cork, Ireland; ${ }^{5}$ Obstetrics and Gynaecology, School of Medicine, University College Dublin, Ireland

\subsection{6/jech-2017-SSMAbstracts. 115}

Background Regular Physical Activity (PA) during pregnancy is associated with a number of health benefits such as preventing pregnancy complications, limiting pregnancy weight gain, and decreasing the risk of gestational diabetes. Despite this, women's PA levels often reduce or cease during pregnancy. Various factors like body mass index, unemployment, and educational levels have been shown to be associated with PA levels during pregnancy. Using the Screening for Pregnancy Endpoint (SCOPE) data, this study aimed to examine the association of health behaviours and psychological well-being on PA levels in pregnancy using the biopsychosocial model.

Methods Nulliparous women with a singleton pregnancy were recruited from a large academic maternity hospital in Cork, Ireland (CUMH) as part of the international SCOPE study. Data was collected at $15 \pm 1$ week's gestation including personal information, lifestyle and psychological measures. The outcome of interest was PA categorised as low, moderate or high levels and covariates were selected using the biopsychosocial model including social (age; years of schooling; socioeconomic status), biological (body mass index), behavioural (diet; smoking) and psychological factors (anxiety; response to pregnancy). Univariate and multivariable multinomial logistic regression examined the association between covariates and PA using Stata IC13.

Results 1774 pregnant women were recruited to SCOPE and women were classified as having low (22.3\%), moderate $(54.4 \%)$ or high $(23.4 \%)$ PA levels $(n=1766)$. In the fully adjusted model, preliminary findings indicate that age categories 30-34 years (OR 2.34 [95\% CI: 1.26-4.37]), $\geq 35$ years (OR 2.06 [95\% CI: 1.01-4.19]) compared to those $<25$ years were positively associated with high PA levels. Having more than 12 years of schooling and a higher socioeconomic status remained significant $(\mathrm{p}<0.05)$ for moderate PA levels. Five portions of fruit and veg a day (OR 1.89 [95\% CI: 1.212.94]), fish consumption (OR 1.47 [95\% CI: 1.07-2.03]) and psychological response to pregnancy (OR 1.04 [95\% CI: 1.01-1.08]) were also positively associated with high levels of PA. Women who reported smoking in their first trimester were $31 \%$ less likely to be in the high physical activity group compared to those who reported no smoking (OR $0.69[95 \%$ 